\title{
CLASSIFICAÇÃO DA CAPACIDADE PRODUTIVA DE POVOAMENTOS DE EUCALIPTO A PARTIR DE PARCELAS TEMPORÁRIAS
}

Débora Jéssica Xavier Gouvea ${ }^{(1)}$; Marcos Vinícius Santana Leite ${ }^{(2)}$; Adriana Leandra Assis $^{(3)}$; Christian Dias Cabacinha ${ }^{(3)}$

(1) Graduada em Engenharia Florestal pela Universidade Federal de Minas Gerais, (deboraxavierpainas@hotmail.com)

(2) Mestrando em Ciências Florestais da Universidade Estadual do Sudoeste da Bahia

(3) Professor Doutor do Instituto de Ciências Agrárias da Universidade Federal de Minas Gerais

Recebido em: 08/04/2016 - Aprovado em: 30/05/2016 - Publicado em: 20/06/2016 DOI: 10.18677/Enciclopedia_Biosfera_2016_028

\begin{abstract}
RESUMO
Objetivou-se com este trabalho realizar a classificação de sítios florestais a partir de uma base de parcelas temporárias. Os dados utilizados no ajuste dos modelos foram provenientes de um povoamento de clones de Eucalyptus urophylla e Eucalyptus grandis localizado no Sul da Bahia, com área de aproximadamente 96.000 ha. Foram utilizados pares de dados de idade e altura média das árvores dominantes de 12.050 parcelas distribuídas em todo o povoamento florestal. Foram testados oito modelos matemáticos amplamente utilizados na literatura florestal para a estimativa da altura dominante: Schumacher, Chapman-Richards, Weibull, SilvaBailey, Prodan, Logístico, Clutter-Jones e Mitscherlich. Os modelos foram avaliados através do erro padrão da estimativa (Syx), dispersão gráfica dos resíduos através do histograma e do gráfico da probabilidade normal dos resíduos, Critério de Informação de Akaike (AIC) e Critério de Informação Bayesiano (BIC). Todos os modelos apresentaram valores estatísticos e gráficos dos resíduos com comportamento semelhante entre si. Foram construídas seis curvas de sítio com amplitude de cinco metros pelo método da curva-guia, utilizando a idade de referência de sete anos. O modelo de Chapman-Richards foi escolhido por apresentar os melhores resultados estatísticos e por ser um modelo biológico. A classe de sítio III apresentou maior representatividade na classificação por talhão, com índice de sítio de 35,5 m.
\end{abstract}

PALAVRAS-CHAVE: Altura dominante, classe de sítio, unidades amostrais.

\section{CLASSIFICATION OF CAPACITY PRODUCTIVE OF Eucalyptus STANDS THROUGH TEMPORARY PLOTS}

\begin{abstract}
The objective of this work was to perform the classification of forest sites from temporary plots. The database used to fit the models were composed by 12,050 records of age and average dominant height obtained from Eucalyptus urophylla and Eucalyptus grandis stands, located in Southern of Bahia, with an area of ENCICLOPÉDIA BIOSFERA, Centro Científico Conhecer - Goiânia, v.13 n.23; p. 3202016
\end{abstract}


approximately 96,000 ha. For this, eight mathematical models were tested: Schumacher, Chapman-Richards, Weibull, Silva-Bailey, Prodan, Logistics, ClutterJones and Mitscherlich. The models were evaluated by the standard error of estimate (Syx), graphic dispersion of residues through the histogram and normal probability of residual plot, Akaike Information Criterion (AIC) and Bayesian Information Criterion (BIC). All models presented statistical values and graphics of residues with a close behavior to each other. Six site curves were constructed, with range of five meters, and the guide curve method using the reference age of seven years. ChapmanRichards' model was chosen, because it showed the best statistical results and is a biological model. The class III site had greater representation in the ranking per field, with a site index of $35.5 \mathrm{~m}$.

KEYWORDS: Dominant height, site class, sampling unit.

\section{INTRODUÇÃO}

O sitio é uma variável que expressa o potencial de produtividade local associada a uma espécie de interesse. Também pode ser compreendido como o potencial de produzir madeira de uma determinada espécie em uma área. Segundo BILA et al. (2012), o estudo da classificação de uma floresta em relação ao potencial de produção é de grande valia, tanto para o melhor manejo dessa área quanto para o seu melhor planejamento, considerando que eles relatam a produtividade de uma floresta. Além do mais, o índice de sítio é uma variável solicitada para o desenvolvimento de modelos de predição da produção florestal. Este ainda possibilita a estratificação por áreas da floresta de acordo com a capacidade produtiva da mesma.

A estimativa do índice de sítio é fundamental na implantação de povoamentos florestais. Um sítio florestal é determinado pela sua qualidade em relação a uma espécie, e é uma classificação da capacidade produtiva através da estimativa de produção de uma espécie em determinada área florestal (SANTOS, 2012). Existem vários métodos para realizar a classificação de sítio, sendo o mais usual o método da construção de curvas de índice local, que classifica de forma quantitativa a capacidade produtiva de cada lugar (CAMPOS \& LEITE, 2013).

Para realizar a classificação de sítios, utilizam-se dados obtidos de análise do tronco, parcelas permanentes ou parcelas temporárias. As parcelas permanentes são a melhor fonte de dados, consistindo em uma rede de parcelas de inventário florestais contínuos. A análise do tronco é outra excelente forma de obtenção de dados, porém se limita à apenas as espécies que apresentarem nitidez nos anéis de crescimento (CAMPOS \& LEITE, 2013).

Segundo SCOLFORO (1997), as parcelas temporárias são muito utilizadas em situações onde não se têm parcelas permanentes e as árvores não apresentam nitidez nos anéis de crescimento. Sendo ainda muito eficientes em levantamentos rápidos e nos inventários pré-cortes. PEGO et al. (2015) utilizaram parcelas temporárias para elaborar a classificação de sítios florestais de uma empresa e conseguiram obter boas estatísticas de ajuste e precisão dos modelos matemáticos e gerar as classes de sítio.

As parcelas temporárias e permanentes são as mais utilizadas para obtenção de dados, porém as permanentes demandam um espaço de tempo muito grande e também um custo mais elevado, enquanto as temporárias apresentam a vantagem de ter um custo operacional reduzido e um levantamento de dados num espaço de tempo mais curto. Pode-se considerar ainda que as parcelas temporárias 
possibilitam trabalhar com informações advindas de inventários convencionais, podendo incorporar novas técnicas de medições, eliminando problemas com erros correlatos e ainda propiciando informações precisas sobre o estoque florestal (SCOLFORO \& MELLO, 2006).

Nesse contexto, este trabalho teve como objetivo realizar a classificação de sítios florestais por talhão, a partir de uma base de dados de parcelas temporárias em um povoamento de eucalipto localizado no Sul da Bahia.

\section{MATERIAL E MÉTODOS}

Os dados utilizados neste estudo foram provenientes de um plantio de clones de Eucalyptus urophylla e Eucalyptus grandis localizado no Sul do estado da Bahia de aproximadamente 96.000 ha de área. O clima é, segundo a classificação de Köppen, Tropical Equatorial (Af): chuvoso, quente e úmido, característico do litoral e Tropical de Monção (Am): quente e úmido, porém com precipitações inferiores ao anterior. A região apresenta temperatura média anual de $24^{\circ} \mathrm{C}$, com pequena amplitude.

Os dados dendrométricos foram oriundos de 12.050 parcelas temporárias de um inventário florestal convencional com idade entre 1,14 a 15,07 anos. Em cada parcela mediu-se: a circunferência a $1,30 \mathrm{~m}$ do solo (CAP) de todas as árvores; altura total das cinco primeiras árvores; e altura total das árvores dominantes, que foram identificadas segundo o conceito de Assmann, correspondendo às três árvores mais grossas da parcela. Foram testados oito modelos matemáticos para o ajuste da altura dominante em função da idade. Os modelos testados são apresentados no quadro 1.

QUADRO 1: Modelos testados para a classificação de sítio.

\begin{tabular}{|c|c|}
\hline Autor & Modelo \\
\hline Schumacher & $\mathrm{Hd}=\beta_{0} \mathrm{e}^{\left(\frac{\beta_{\mathrm{A}}}{\mathrm{Id}}\right)}$ \\
\hline Chapman-Richards & $\mathrm{Hd}=\beta_{0} \mathrm{e}^{\left(-\beta_{1} I d\right)^{\beta_{2}}}$ \\
\hline Weibull & $\mathrm{Hd}=\beta_{0}-\beta_{1} e^{\left(\beta_{2} I d^{\beta}\right)}$ \\
\hline Silva-Bailey & $\mathrm{Hd}=\beta_{0} \mathrm{e}\left(\beta_{1} \beta_{2}^{\mathrm{ld}}\right)$ \\
\hline Mitscherlich & $\mathrm{Hd}=\beta_{0}-\beta_{1} \beta_{2}^{\mathrm{Id}}$ \\
\hline Clutter-Jones & $\mathrm{Hd}=\beta_{0}\left(1+\beta_{1} \mathrm{Id}^{\beta_{2}}\right)^{\beta_{3}}$ \\
\hline Prodan & $\mathrm{Hd}=\mathrm{Id}^{2} /\left(\beta_{0}+\beta_{1} \mathrm{Id}+\beta_{2} \mathrm{Id}^{2}\right)$ \\
\hline Logístico & $\mathrm{Hd}=\beta_{0} /\left[1+\beta_{1} \mathrm{e}^{\left(-\beta_{2} \mathrm{Id}\right)}\right]$ \\
\hline
\end{tabular}

Fonte: Adaptado de FELDE et al. (2010) e NASCIMENTO et al. (2015). Onde: $\mathrm{Hd}=$ altura dominante $(\mathrm{m})$; Id= idade (anos); $\beta_{i}=$ coeficientes estimados; $e=$ exponencial. 
Os ajustes dos modelos matemáticos foram realizados no software $R$ versão 3.2.2, ajustados por meio de regressão não linear através do algoritmo de Levenberg Marquardt com o auxílio do packages "minpack.Im". Foram analisados os seguintes critérios estatísticos: erro padrão da estimativa (Syx), dispersão gráfica dos resíduos através do histograma e do gráfico da probabilidade normal dos resíduos, critério de informação de Akaike (AIC) e critério de informação Bayesiano (BIC).

Para a construção das curvas de sítio, o Microsoft Excel e o método da curva guia foram utilizados, gerando curvas anamórficas. Calcularam-se os fatores através da razão entre altura dominante observada e altura dominante estimada e, a partir deles, foram determinados os limites inferiores e superiores dos sítios florestais, com uma amplitude de $5 \mathrm{~m}$. Em seguida, foi realizada a classificação para cada parcela e geradas as curvas referentes às seis classes de sítio. Após a classificação no nível de parcela, realizou-se a classificação no nível de talhão. Dessa forma, a classe de um talhão foi determinada pela maior quantidade de parcelas pertencentes a uma classe. Assim, se houvesse a quantidade semelhante de parcelas pertencentes a duas classes diferentes, escolhia-se a parcela com produtividade inferior.

\section{RESULTADOS E DISCUSSÃO}

$\mathrm{Na}$ Tabela 1 encontram-se as estatísticas e os coeficientes de ajustes dos modelos testados.

TABELA 1: Coeficientes e estatísticas dos modelos ajustados.

\begin{tabular}{ccccccccc}
\hline \multirow{2}{*}{ Modelos } & \multicolumn{3}{c}{ Coeficientes da regressão } & \multicolumn{4}{c}{ Estatísticas } \\
\cline { 2 - 9 } & $\boldsymbol{\beta}_{\mathbf{0}}$ & $\boldsymbol{\beta}_{\mathbf{1}}$ & $\boldsymbol{\beta}_{\mathbf{2}}$ & $\boldsymbol{\beta}_{\mathbf{3}}$ & Syx $(\mathbf{m})$ & Syx (\%) & AlC & BIC \\
\hline Schumacher & 45,6432 & 2,2803 & & - & 2,486 & 8,70 & $56.149,16$ & $56.171,35$ \\
Chapman e Richards & 39,7047 & 0,2569 & 1,0003 & - & 2,470 & 8,64 & $55.993,10$ & $56.022,69$ \\
Weibull & 4,1077 & $-45,0811$ & $-2,5565$ & $-0,8972$ & 2,473 & 8,65 & $56.021,38$ & $56.058,36$ \\
Silva-Bailey & 38,4068 & $-1,7321$ & 0,7007 & - & 2,481 & 8,68 & $56.104,20$ & $56.133,79$ \\
Mitscherlich & 39,6337 & 39,8690 & 0,7718 & - & 2,470 & 8,64 & $55.992,62$ & $56.022,21$ \\
Clutter-Jones & 44,6730 & 6,0911 & $-1,3773$ & $-0,8657$ & 2,472 & 8,65 & $56.012,12$ & $56.049,10$ \\
Prodan & 0,0569 & 0,0583 & 0,0208 & - & 2,472 & 8,65 & $56.014,86$ & $56.044,45$ \\
Logístico & 37,6789 & 3,0339 & 0,4517 & - & 2,498 & 8,74 & $56.260,24$ & $56.289,82$ \\
\hline
\end{tabular}

De acordo com a análise estatística (Tabela 2), todos os modelos apresentaram a mesma tendência de ajuste. Os valores de Syx foram próximos aos encontrados por NASCIMENTO et al. (2015). Os melhores valores de Syx (\%) foram observados para os modelos de Chapman-Richards e Mitscherlich, 8,64\%, e o pior para o modelo Logístico, 8,74\%. RETSLAFF et al. (2015) também verificaram que modelos de Chapman-Richards e Mitscherlich apresentaram as melhores estatísticas. Nos critérios AIC e BIC os modelos de Chapman-Richards e Mitscherlich demonstraram os melhores valores e a superioridade em relação aos demais modelos.

A Figura 1 apresenta os histogramas de frequência dos resíduos para os modelos testados. Observa-se uma maior frequência dos resíduos nas classes centrais, o que era esperado, pois nas estimativas de altura dominantes tem uma ocorrência maior de menores erros (classes centrais) e uma menor frequência é observada de maiores erros (classes periféricas), ou seja, os modelos conseguem estimar as alturas das árvores dominantes com pouca tendência em sub ou superestimar os valores. 


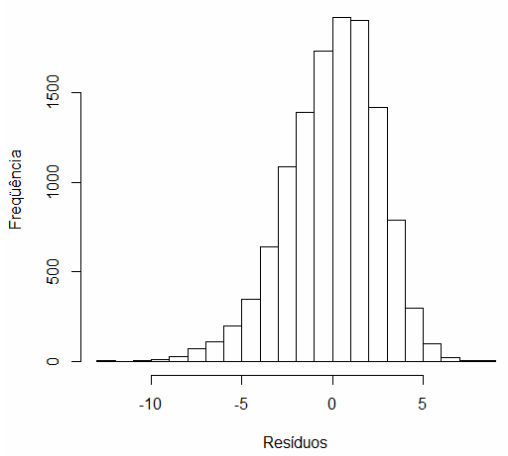

(d)

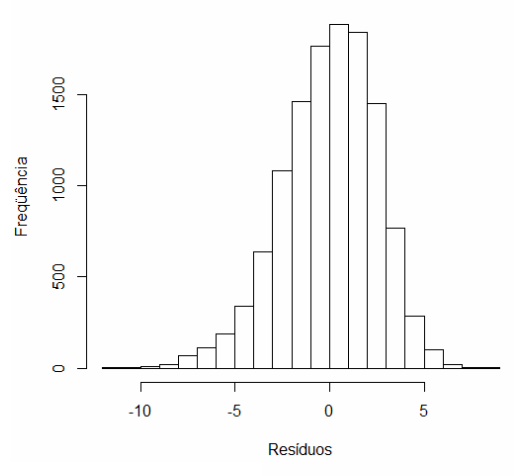

(g) (b)

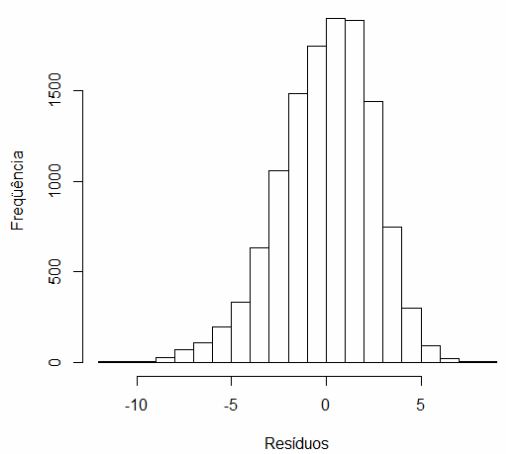

(e)

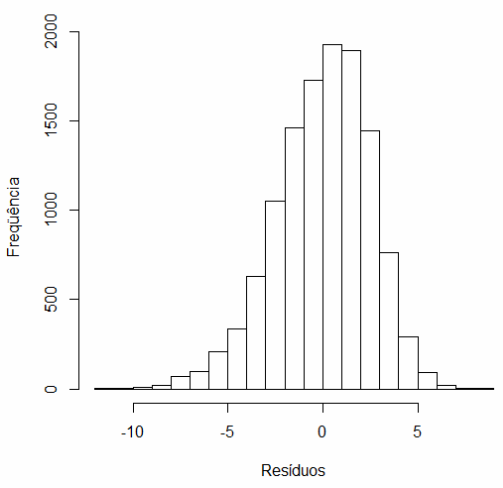

(c)

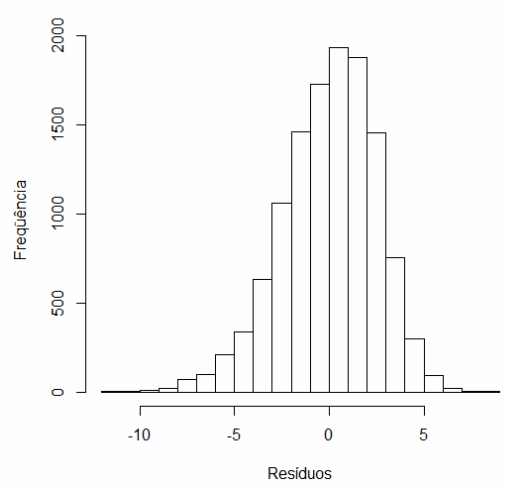

(f)

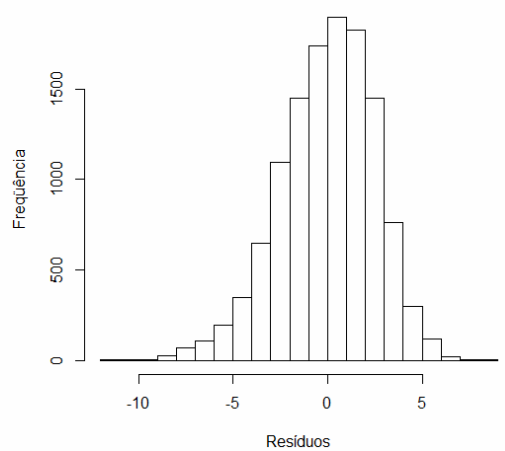

(h)
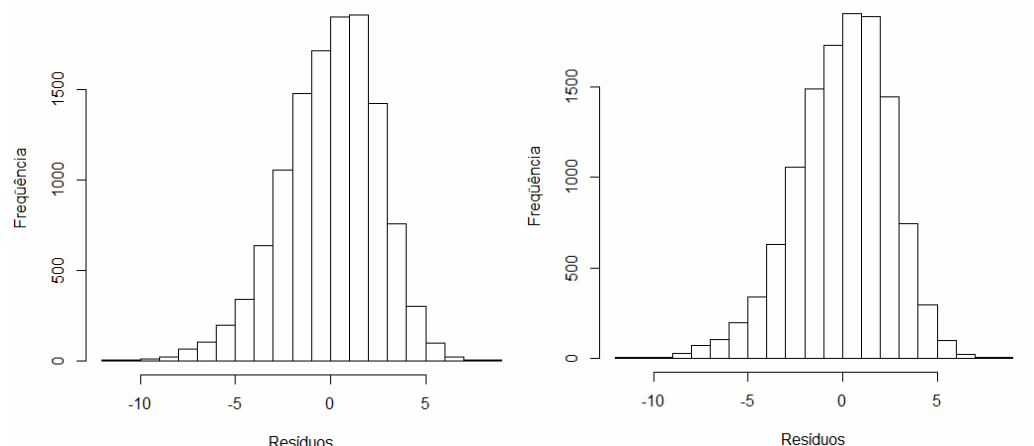

FIGURA 1- Histograma de frequência para os modelos testados, sendo: a) Schumacher, b) Chapman e Richards, c) Weibull, d) Silva-Bailey, e) Mitscherlich, f) Clutter-Jones, g) Prodan e h) Logístico.

A Figura 2 apresenta os gráficos de probabilidade normal dos resíduos para os modelos testados. Observa-se que os resíduos se distribuem homogeneamente ao longo dos valores ajustados, confirmando então a capacidade dos modelos em realizarem estimativas sem tendenciosidade. 
(a)

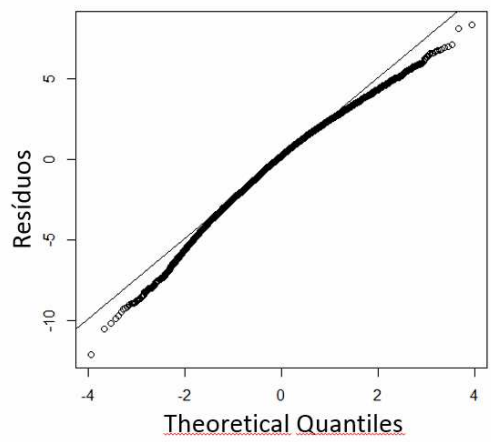

(d)

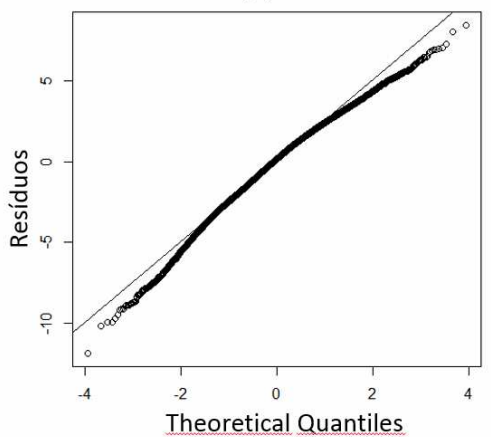

(b)

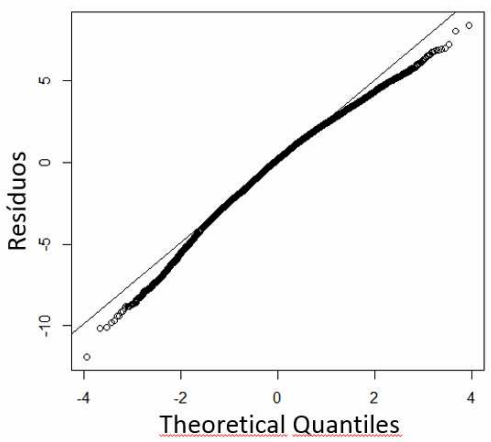

(e)

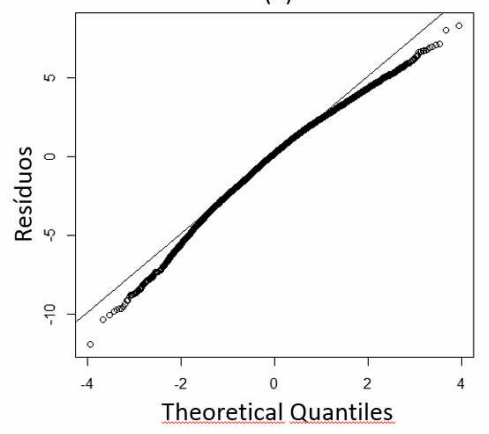

(g)

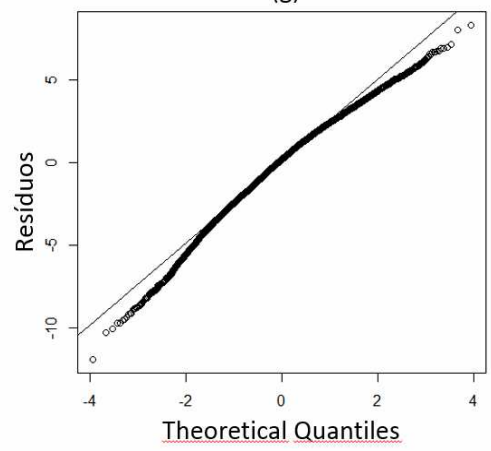

(c)

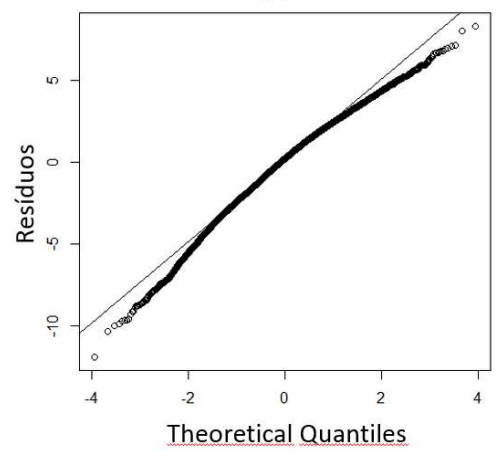

(f)

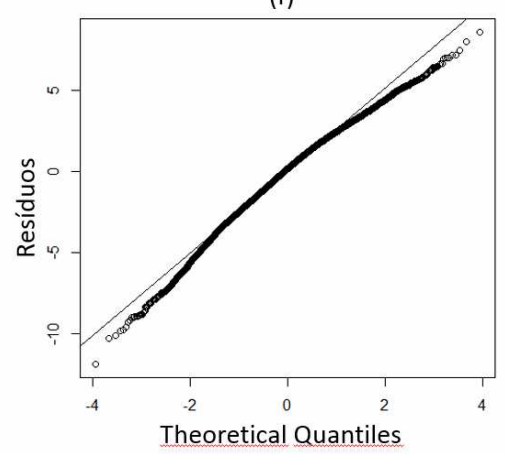

(h)

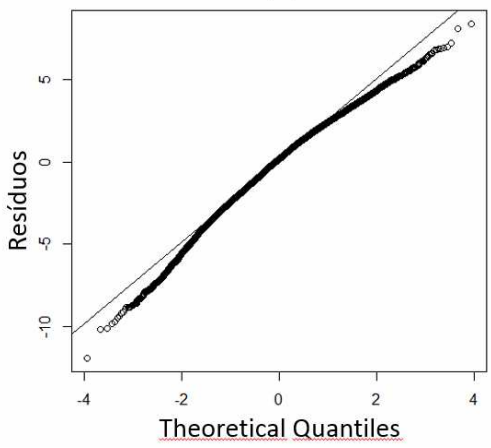

FIGURA 2- Gráficos de probabilidade normal dos resíduos para os modelos testados, sendo: a) Schumacher, b) Chapman e Richards, c) Weibull, d) Silva-Bailey, e) Mitscherlich, f) Clutter-Jones, g) Prodan e h) Logístico.

Pode-se observar nas Figuras 1 e 2 uma grande proximidade dos gráficos de resíduos entre os modelos testados. Foi possível verificar que os modelos apresentam uma leve tendência em superestimar a altura dominante nas idades iniciais, porém, apresentaram normalidade e resíduos homogeneamente distribuídos ao longo dos valores ajustados indicando um bom ajuste para todos os modelos testados.

Os modelos apresentaram boas estatísticas e precisão no ajuste aceitáveis, sendo considerados adequados para gerar as curvas de sítio, pois não apresentaram tendenciosidade em seus gráficos dos resíduos e o erro padrão da estimativa (Syx) foram muito próximos, ou seja, houve uma boa representatividade dos dados em diferentes idades.

O modelo de Chapman-Richards foi o selecionado, pois apresentou bons valores estatísticos, sendo um modelo biológico e tradicionalmente empregado na 
classificação de sítios. RETSLAFF et al. (2015) escolheram o modelo de ChapmanRichards, pois apresentou maior precisão no ajuste, bem como melhores valores estatísticos. Esse modelo também foi selecionado por FELDE et al. (2010) e por HREÇAY et al. (2014).

Foram construídas as curvas de sítio para o modelo escolhido e, para isso, foi necessário determinar o número de curvas de sítio para representar os resultados do crescimento em altura. As diferenças no padrão de crescimento em altura apontaram a necessidade de seis curvas de sítio com amplitude de cinco metros, cujos valores são apresentados na Tabela 2. Dessa forma, as curvas englobaram todos os valores de altura dominante amostrada nas parcelas. A idade de referência escolhida foi a de sete anos, por ser a idade de rotação do povoamento.

TABELA 2: Classes de sítio, intervalos de classes e os seus respectivos valores de Índice de sítio.

\begin{tabular}{ccc}
\hline Classes de sítio & Intervalos de classe & Índice de sítio \\
\hline I & $43 \mathrm{I}---48 \mathrm{~m}$ & 45,5 \\
II & $38 \mathrm{I}--43 \mathrm{~m}$ & 40,5 \\
III & $33 \mathrm{I}---38 \mathrm{~m}$ & 35,5 \\
IV & $28 \mathrm{I}--\mathrm{3} 3 \mathrm{~m}$ & 30,5 \\
V & $23 \mathrm{I}---28 \mathrm{~m}$ & 25,5 \\
VI & $18 \mathrm{I}---23 \mathrm{~m}$ & 20,5 \\
\hline
\end{tabular}

$\mathrm{Na}$ Figura 2 são apresentadas as curvas construídas para o modelo escolhido. O modelo de Chapman-Richards se ajustou bem aos dados e as curvas conseguiram abranger todos os dados de altura dominante em diferentes idades.

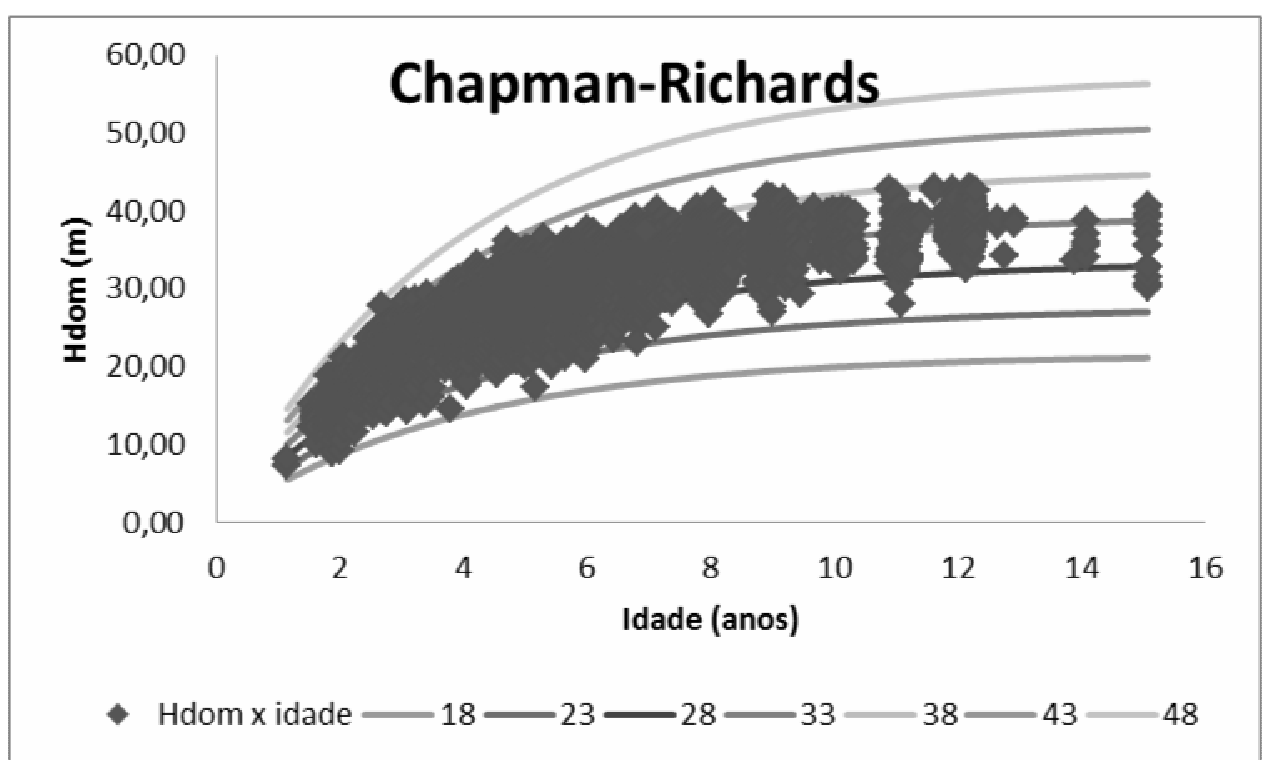

FIGURA 2: Curvas de sítio geradas pelo modelo de Chapman-Richards.

A Tabela 3 expressa a classificação de sítios a nível de parcelas e talhões para o modelo escolhido. A maioria das parcelas e dos talhões se concentraram nas duas classes centrais III e IV, apresentando 6.267 e 4.847 parcelas, e para talhões 
foram observados 3.593 e 2.944 , respectivamente. Isso pode ter ocorrido por serem parcelas com árvores mais velhas, onde seu crescimento já esteja estabilizado. À medida que se dirige para os limites inferior e superior encontra-se uma menor representatividade na classificação, como observado na classe VI com 17 parcelas e 10 talhões. Já a classe I apresentou a menor representatividade, com apenas 4 parcelas e 3 talhões. Observou que a classe I, sendo a mais produtiva, encontravam parcelas em idades mais jovens, cujo desenvolvimento foi mais vigoroso e o que as fez ser mais produtivas.

Segundo SILVA et al. (2013), as parcelas que se encontram próximas aos limites das classes de sítio podem flutuar de uma classe para a outra. Isso ocorre devido às mudanças climáticas ou à fatores do povoamento que possam interferir no desenvolvimento das árvores. Ainda segundo estes autores, à medida que o povoamento envelhece, menos frequentes são essas mudanças, pois menores são as variações do padrão de desenvolvimento, o que mantem a altura dominante mais estável.

TABELA 3: Número de parcelas e talhões em cada classe de sítio

\begin{tabular}{ccccccc}
\hline & \multicolumn{7}{c}{ Classe de sítio } \\
\hline & I & II & III & IV & V & VI \\
\cline { 2 - 7 } Parcelas & 4 & 352 & 6267 & 4847 & 563 & 17 \\
Talhões & 3 & 185 & 3593 & 2944 & 294 & 10 \\
\hline
\end{tabular}

\section{CONCLUSÃO}

O modelo matemático de Chapman-Richards foi selecionado para a classificação de sítios florestais a partir de uma base de parcelas temporárias, pois é um modelo biológico que apresenta bons valores estatísticos e por ser tradicionalmente empregado na classificação de sítios.

A classe de sítio com maior representatividade na classificação por talhão é a classe III, permitindo inferir que o povoamento florestal, localizado no Sul da Bahia, apresenta índice de sítio de $35,5 \mathrm{~m}$ como média de altura para as condições estudadas.

\section{REFERÊNCIAS}

BILA, J. M.; SANQUETTA, C. R.; MACHADO, S. A. Classificação de sítios com base em fatores edáficos para Pinus caribaea var. hondurensis na região de Prata, Minas Gerais. Revista Floresta, Curitiba, v. 42, n.3, p. 465-474, 2012.

CAMPOS, J. C. C.; LEITE, H. G. Mensuração floresta: perguntas e respostas. 4 ed. Viçosa: Editora UFV, 2013. 605 p.

FELDE, J. L.; RETSLAFF, F. A. S.; FIGUEIREDO FILHO, A.; DIAS A. N. Curvas de índice de sítio para povoamentos de Eucalyptus dunnii na região central do Paraná. Anais do II Seminário de Atualização Florestal e XI Semana de Estudos Florestais, Irati, 2010.

HREÇAY, L.; WEBER, V. P.; SILVA, L. T. M. da; LIZ, J. P. C. de; VANDRESEN, P. B. Curvas de índice de sítio para plantios florestais comerciais de Pinus taeda L. 
localizados na região oeste catarinense. $3^{\circ}$ Encontro brasileiro de silvicultura, Campinas, 2014.

NASCIMENTO, F. A. F. do; DIAS, A. N.; FIGUEIREDO FILHO, A.; MIRANDA, G. de M.; ARCE, J. E. Sistema de crescimento e produção para povoamentos de Pinus taeda na região norte de Santa Catarina. Revista Cerne, Lavras, v. 21 n. 2, p. 235242, 2015. Disponível em: <http://dx.doi.org/10.1590/01047760201521021494>. doi: $10.1590 / 01047760201521021494$

PEGO, M. F. F.; ASSIS, A. L. de; CABACINHA, C. D. Classificação de sítios florestais em povoamentos de eucalipto na microrregião de Salinas, Minas Gerais. Enciclopédia Biosfera, Goiânia, v. 11, n. 21, p. 534-542, 2015.

RETSLAFF, F. A. S.; FIGUEIREDO FILHO, A.; DIAS, A. N.; BERNETT, L. G.; FIGURA, M. A. Curvas de sítio e relações hipsométricas para Eucalyptus grandis na região dos campos gerais, Paraná. Revista Cerne, Lavras, v.21, n.2, p. 219-225, 2015. Disponível em: <http://dx.doi.org/10.1590/01047760201521021349>. doi: 10.1590/01047760201521021349

SANTOS, R. C. Curvas de crescimento em altura e índice de sítio de povoamentos clonais de Eucalyptus spp. na Chapada do Araripe, Pernambuco. 2012. 83 f. Dissertação (Pós-Graduação e Ciências Florestais) Universidade Federal Rural de Pernambuco, Recife, 2012.

SCOLFORO, J. R. S. Biométria florestal - módulo 3: métodos para a classificação de sítios florestais. Lavras. 1997, 151 p.

SCOLFORO, J. R. S.; MELLO, J. M. de. Inventário florestal. Lavras. 2006, 561 p.

SILVA, F. da; PIMENTEL, A.; CORTE, A. P. D.; SANQUETTA, C. R. Classificação de sítio para Pinus caribaea var. hondurensis na região do Triângulo Mineiro. Revista Acadêmica, Ciências Agrárias Ambiental, Curitiba, v. 11, Supl. 1, p. S105-S112, 2013. 